\title{
Azimuthally Polarized Spatial Dark Solitons: Exact Solutions of Maxwell's Equations in a Kerr Medium
}

\author{
Alessandro Ciattoni* \\ Istituto Nazionale per la Fisica della Materia, UdR Universitá dell'Aquila, 67010, L'Aquila, Italy \\ and Dipartimento di Fisica, Universitá dell'Aquila, 67010 L'Aquila, Italy \\ Bruno Crosignani \\ Dipartimento di Fisica, Universitá dell'Aquila, 67010 L'Aquila, Italy, \\ Istituto Nazionale di Fisica della Materia, UdR Roma "La Sapienza", 00185 Roma, Italy, \\ and California Institute of Technology 128-95, Pasadena, California 91125, USA \\ Paolo Di Porto \\ Dipartimento di Fisica, Universitá dell'Aquila, 67010, L'Aquila, Italy \\ and Istituto Nazionale di Fisica della Materia, UdR Roma "La Sapienza", 00185 Roma, Italy
}

Amnon Yariv

California Institute of Technology 128-95, Pasadena, California 91125, USA

(Received 27 September 2004; published 23 February 2005)

\begin{abstract}
Spatial Kerr solitons, typically associated with the standard paraxial nonlinear Schrödinger equation, are shown to exist to all nonparaxial orders as exact solutions of Maxwell's equations in the presence of the vectorial Kerr effect. More precisely, we prove the existence of azimuthally polarized, spatial, dark soliton solutions of Maxwell's equations, while exact linearly polarized $(2+1) \mathrm{D}$ solitons do not exist. Our $a b$ initio approach predicts the existence of dark solitons up to an upper value of the maximum field amplitude, corresponding to a minimum soliton width of about one-fourth of the wavelength.
\end{abstract}

DOI: 10.1103/PhysRevLett.94.073902

PACS numbers: 42.65.Tg, 42.81.Dp

Bright and dark optical spatial solitons have been and still are the object of an intense theoretical and experimental investigation. [1,2] In particular, Kerr solitons have always represented the theoretical reference model in view of the simplicity of their analytic description. This is mainly due to the fact that they are the solution of an equation, the so-called nonlinear Schrödinger equation (NLS), which is exactly integrable and also admits, in particular cases, of simple analytic solutions. The main limit of the NLS is that it is only able to describe scalar optical propagation in the paraxial approximation. Nonparaxial descriptions have been introduced by adopting suitable asymptotic expansions in the smallness parameter $\lambda / d$ (where $d$ is the beam width) which result in improved versions of NLS including both scalar [3-6] and vectorial [7-10] contributions. Besides eliminating the unphysical catastrophic collapse associated with standard NLS [11-13], they show that the transverse Cartesian components of $(2+1) \mathrm{D}$ propagating beams are mutually coupled, so that linearly polarized $(2+1) \mathrm{D}$ Kerr solitons cannot exist. The same nonparaxial approach can also be used to predict the existence of $(1+1) \mathrm{D}$ bright and dark solitons [14,15], to the first significant order in $\lambda / d$.

The standard or improved versions of the NLS are limited by the underlying approximation scheme and fail whenever $\lambda / d$ approaches one. The question naturally arises: Are there solitons to all nonparaxial orders? More in general, is it possible to find exact soliton solutions of Maxwell's equations?

In this Letter we find, by starting from Maxwell's equations, that an exact solution (to all nonparaxial orders) indeed exists in the form of an azimuthally polarized dark soliton, i.e.,

$$
\mathbf{E}(r, \varphi, z)=e^{i \chi z} E(r) \hat{\mathbf{e}}_{\varphi}
$$

where $r, \varphi, z$ are cylindrical coordinates with unit vectors $\hat{\mathbf{e}}_{r}, \hat{\mathbf{e}}_{\varphi}, \hat{\mathbf{e}}_{z}$ and $\chi$ is a real number. More precisely, we are able to prove the existence of azimuthally polarized dark solitons, i.e., of fields of the kind given in Eq. (1), where $E(r)$ monotonically ranges from $E(0)=0$ to $E(\infty)=E_{\infty}$ and $E_{\infty}$ is a given real constant. We numerically evaluate the soliton shape and its existence curve, our results being obtained without any approximation, so that the whole range of possible soliton widths is considered without any formal distinction between paraxial and nonparaxial regimes. We note that the existence of azimuthally polarized, circularly symmetric fields of the form $E(r, z) \hat{\mathbf{e}}_{\varphi}$, of which the soliton given in Eq. (1) is a specific case, is due to the simple symmetry properties of Kerr nonlinearity.

A monochromatic electromagnetic field $\operatorname{Re}[\mathbf{E} \exp (-i \omega t)], \operatorname{Re}[\mathbf{B} \exp (-i \omega t)]$ propagating in a nonlinear medium obeys Maxwell's equations

$$
\nabla \times \mathbf{E}=i \omega \mathbf{B}, \quad \nabla \times \mathbf{B}=-i \frac{\omega}{c^{2}} n_{0}^{2} \mathbf{E}-i \omega \mu_{0} \mathbf{P}_{n l},
$$


where $n_{0}$ labels the linear refractive index and $\mathbf{P}_{n l}$ is the nonlinear polarizability. In the case of nonresonant isotropic media, the vectorial Kerr effect is described by the polarizability [16]

$$
\mathbf{P}_{n l}=\frac{4}{3} \epsilon_{0} n_{0} n_{2}\left[|\mathbf{E}|^{2} \mathbf{E}+\frac{1}{2}(\mathbf{E} \cdot \mathbf{E}) \mathbf{E}^{*}\right],
$$

$n_{2}$ being the nonlinear refractive index coefficient. After eliminating $\mathbf{B}$ from Eq. (2) we get

$$
\nabla \times \nabla \times \mathbf{E}=k^{2} \mathbf{E}+k^{2} \frac{4}{3} \frac{n_{2}}{n_{0}}\left[|\mathbf{E}|^{2} \mathbf{E}+\frac{1}{2}(\mathbf{E} \cdot \mathbf{E}) \mathbf{E}^{*}\right]
$$

where $k=n_{0} \omega / c$. Let us consider fields of the form

$$
\mathbf{E}(r, \varphi, z)=E_{\varphi}(r, z) \hat{\mathbf{e}}_{\varphi}+E_{z}(r, z) \hat{\mathbf{e}}_{z},
$$

describing a circularly symmetric field with vanishing radial component. Inserting Eq. (5) in Eq. (4), we obtain

$$
\begin{aligned}
\frac{\partial^{2} E_{z}}{\partial r \partial z}= & 0, \\
\frac{\partial^{2} E_{\varphi}}{\partial z^{2}}+\frac{\partial}{\partial r}\left(\frac{\partial E_{\varphi}}{\partial r}+\frac{E_{\varphi}}{r}\right)= & -k^{2} E_{\varphi}-k^{2} \frac{4}{3} \frac{n_{2}}{n_{0}} \\
& \times\left[|\mathbf{E}|^{2} E_{\varphi}+\frac{1}{2}(\mathbf{E} \cdot \mathbf{E}) E_{\varphi}^{*}\right], \\
\frac{\partial^{2} E_{z}}{\partial r^{2}}+\frac{1}{r} \frac{\partial E_{z}}{\partial r}= & -k^{2} E_{z}-k^{2} \frac{4}{3} \frac{n_{2}}{n_{0}} \\
& \times\left[|\mathbf{E}|^{2} E_{z}+\frac{1}{2}(\mathbf{E} \cdot \mathbf{E}) E_{z}^{*}\right] .
\end{aligned}
$$

Internal consistency of the set of Eqs. (6) (three equations in two unknowns) requires $E_{z}=0$. As a consequence, the second of Eqs. (6) yields

$$
\frac{\partial^{2} E_{\varphi}}{\partial z^{2}}+\frac{\partial}{\partial r}\left(\frac{\partial E_{\varphi}}{\partial r}+\frac{E_{\varphi}}{r}\right)=-k^{2} E_{\varphi}-2 k^{2} \frac{n_{2}}{n_{0}}\left|E_{\varphi}\right|^{2} E_{\varphi} .
$$

We note that circular symmetry and polarization imposed to the field, together with the symmetry properties of Kerr effect, have allowed us to reduce Maxwell's equations to the single Eq. (7). Equation (7) is conveniently rewritten in the dimensionless form

$$
\frac{\partial^{2} U}{\partial \zeta^{2}}+2 \frac{\partial}{\partial \rho}\left(\frac{\partial U}{\partial \rho}+\frac{U}{\rho}\right)=-U-2 \gamma|U|^{2} U,
$$

where $\rho=\sqrt{2} k r, \zeta=k z, U=\sqrt{\left|n_{2}\right| / n_{0}} E_{\varphi}$, and $\gamma=$ $n_{2} /\left|n_{2}\right|$. It is important to stress that Eq. (8) has been derived from Maxwell's equations without any approximation and, as a consequence, it describes propagation to any order of nonparaxiality. If we look for soliton solutions of the form

$$
U(\rho, \zeta)=e^{i \alpha \zeta} u(\rho)
$$

where [see Eq. (1)] $\alpha=\chi / k$, Eq. (8) becomes

$$
\frac{d}{d \rho}\left(\frac{d u}{d \rho}+\frac{u}{\rho}\right)=\frac{1}{2}\left(\alpha^{2}-1\right) u-\gamma u^{3} .
$$

Both the structure of Eq. (10) and the azimuthal field polarization dictate $u(0)=0$, so that azimuthally polarized bright solitons do not exist. In order to find dark solitons, we introduce the further condition

$$
\lim _{\rho \rightarrow \infty} u(\rho)=u_{\infty}
$$

together with the vanishing of all derivatives for $\rho \rightarrow \infty$. Since focusing media $\left(\gamma=1\right.$, i.e., $\left.n_{2}>0\right)$ are not able to support dark solitons, we consider hereafter defocusing media ( $\gamma=-1$, i.e., $n_{2}<0$ ), so that Eq. (10) reads

$$
\frac{d}{d \rho}\left(\frac{d u}{d \rho}+\frac{u}{\rho}\right)=\frac{1}{2}\left(\alpha^{2}-1\right) u+u^{3},
$$

which implies, together with the above boundary condition at infinity,

$$
\alpha= \pm \sqrt{1-2 u_{\infty}^{2}} .
$$

While positive and negative signs of $\alpha$ respectively refer to forward and backward travelling solitons [see Eq. (9)], $u(\rho)$ depends on $\alpha^{2}$ [see Eq. (10)] and is clearly the same in the two cases. Equation (13) shows the existence of an upper threshold for the soliton asymptotic amplitude

$$
u_{\infty}<\frac{1}{\sqrt{2}}
$$

since, otherwise, $\alpha$ would become imaginary. From an intuitive point of view, the existence of this threshold is related to the dominant defocusing effect due to the nonlinearity over the focusing one due to diffraction. If we now insert Eq. (13) into Eq. (12), we obtain

$$
\frac{d}{d \rho}\left(\frac{d u}{d \rho}+\frac{u}{\rho}\right)=\left(u^{2}-u_{\infty}^{2}\right) u .
$$

We have carried out a numerical integration of Eq. (15) with boundary conditions $u(0)=0$ and $u(\infty)=u_{\infty}$, by employing a standard shooting-relaxation method for boundary value problems. The results of our simulations confirm the existence of dark solitons in the range of field amplitudes $0<u_{\infty}<1 / \sqrt{2}$. Different soliton profiles are reported in Fig. 1. We have investigated, by using Eq. (8), the stability of our soliton by testing propagation of a perturbed soliton profile over several diffraction lengths. This analysis, performed over a number of situations, furnishes a positive test for the overall robustness of our solution. In particular, in Fig. 2 we report the evolution of a numerical soliton corresponding to $u_{\infty}=0.2$ while, in Fig. 3, we report a typical numerical simulation for a boundary condition at $\zeta=0$ resulting from the superposition of the soliton and of a Gaussian perturbation of amplitude one-tenth of $u_{\infty}$. 


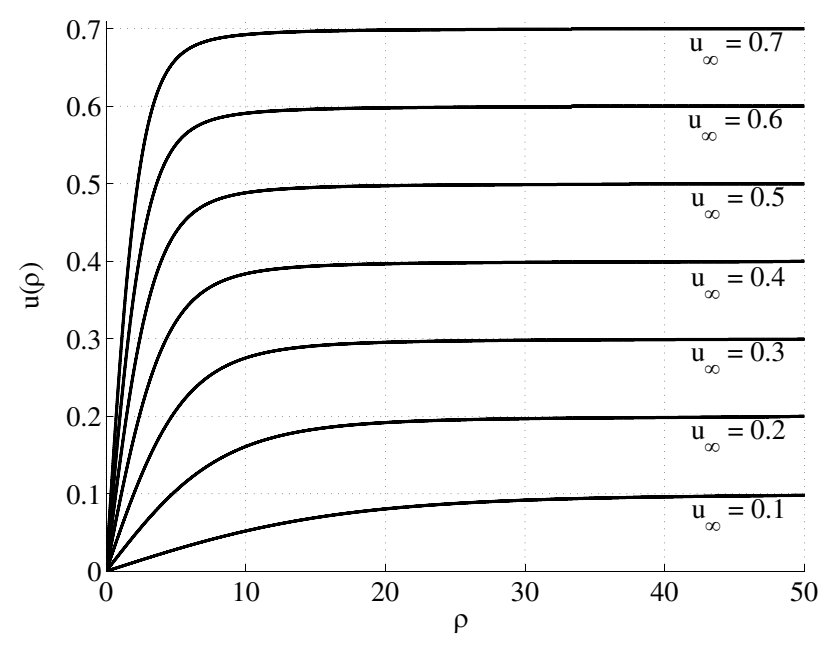

FIG. 1. Soliton profile $u(\rho)$ for various values of $u_{\infty}$.

In order to complete our analysis, we now evaluate both the magnetic field and the Poynting vector. Recalling the expression of the soliton electric field

$$
\mathbf{E}=\sqrt{\frac{n_{0}}{\left|n_{2}\right|}} e^{i \alpha k z} u(\sqrt{2} k r) \hat{\mathbf{e}}_{\varphi},
$$

we obtain, from the first of Eqs. (2) written in cylindrical coordinates,

$$
\mathbf{B}=-\sqrt{\frac{n_{0}}{\left|n_{2}\right|}} e^{i \alpha k z} \frac{k}{\omega}\left[\alpha u \hat{\mathbf{e}}_{r}+i \sqrt{2}\left(\frac{d u}{d \rho}+\frac{u}{\rho}\right) \hat{\mathbf{e}}_{z}\right]_{\rho=\sqrt{2} k r} .
$$

The magnetic field has a radial component whose shape coincides with that of the electric field, and a vanishing azimuthal component, so that $\mathbf{E}$ and $\mathbf{B}$ are mutually orthogonal. With the help of Eqs. (16) and (17), the time

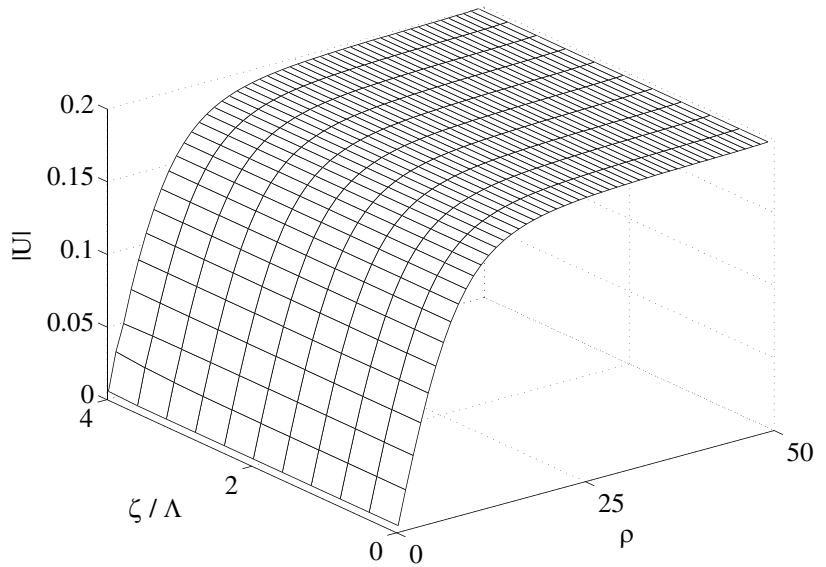

FIG. 2. Amplitude $|U|$ of the numerical solution of Eq. (8) with boundary conditions corresponding to the soliton profile characterized by $u_{\infty}=0.2$ (see Fig. 1). The longitudinal coordinate ranges from zero to four dimensionless diffraction lengths $\Lambda=\Delta^{2}, \Delta$ being the soliton dimensionless HWHM. averaged Poynting vector

$$
\mathbf{S}=\frac{1}{2 \mu_{0}} \operatorname{Re}\left(\mathbf{E} \times \mathbf{B}^{*}\right)
$$

turns out to be given by

$$
\mathbf{S}(r)=\frac{\alpha k}{2 \omega \mu_{0}} \frac{n_{0}}{\left|n_{2}\right|} u^{2}(\sqrt{2} k r) \hat{\mathbf{e}}_{z}=\frac{\alpha k}{2 \omega \mu_{0}}|\mathbf{E}|^{2} \hat{\mathbf{e}}_{z} .
$$

We note that $\mathbf{S}$ is parallel to the $z$ axis, consistently with the shape-invariant nature of solitons. From an analytical point of view, this corresponds to the $\pi / 2$ phase difference between $B_{z}$ and $E_{\varphi}$ [see Eqs. (16) and (17)]. As expected, the Poynting vector is either parallel or antiparallel to $\hat{\mathbf{e}}_{z}$ according to the sign of $\alpha$, while its amplitude is proportional to $|\mathbf{E}|^{2}$. The above plane wavelike properties are consistent with the nondiffractive nature of exact solitons.

It is worthwhile to underline that, in the case of the azimuthal dark solitons we are considering, the asymptotic optical intensity $I_{\infty}=|\mathbf{S}(\infty)|$ turns out not to be proportional to $u_{\infty}^{2}$. In fact, by using Eqs. (13) and (19), one obtains

$$
I_{\infty}\left(u_{\infty}\right)=I_{0} u_{\infty}^{2} \sqrt{1-2 u_{\infty}^{2}}
$$

where $I_{0}=k n_{0} /\left(2 \omega \mu_{0}\left|n_{2}\right|\right)$. Equation (20) shows that the asymptotic optical intensity is not a monotonically increasing function of the asymptotic field amplitude, but reaches its maximum threshold value $I_{\infty}^{\max }=I_{0} / 3^{3 / 2}$ in correspondence to $u_{\infty}=1 / \sqrt{3}$. This is connected to the $\alpha$ dependence of the magnetic field [see Eq. (17)] whose radial part tends to vanish for $u_{\infty} \rightarrow 1 / \sqrt{2}$. A related and relevant consequence of Eq. (20) is the existence of two solitons of different widths for a given asymptotic optical intensity. The existence curve relating the normalized half width at half maximum (HWHM) of the soliton optical intensity profile $|\mathbf{S}(\rho)|$ to $u_{\infty}$ is reported in Fig. 4. In particular, Fig. 4

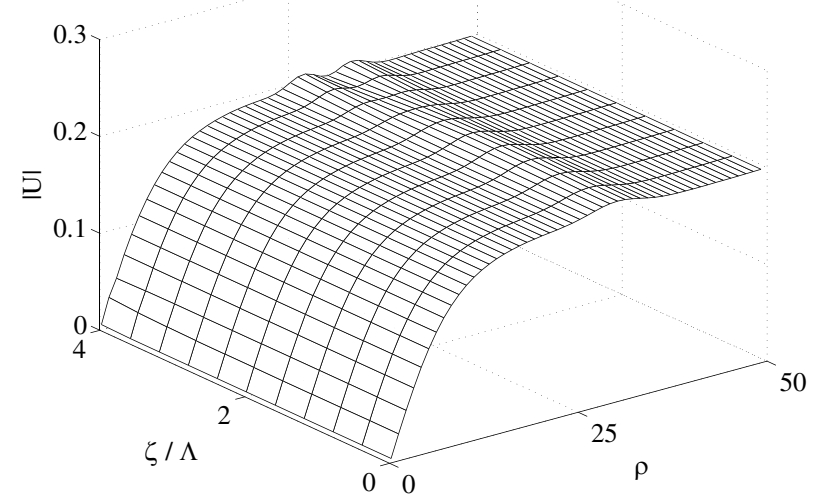

FIG. 3. Amplitude $|U|$ of the numerical solution of Eq. (8) with boundary conditions corresponding to the soliton of Fig. 2 perturbed at $\zeta=0$. 


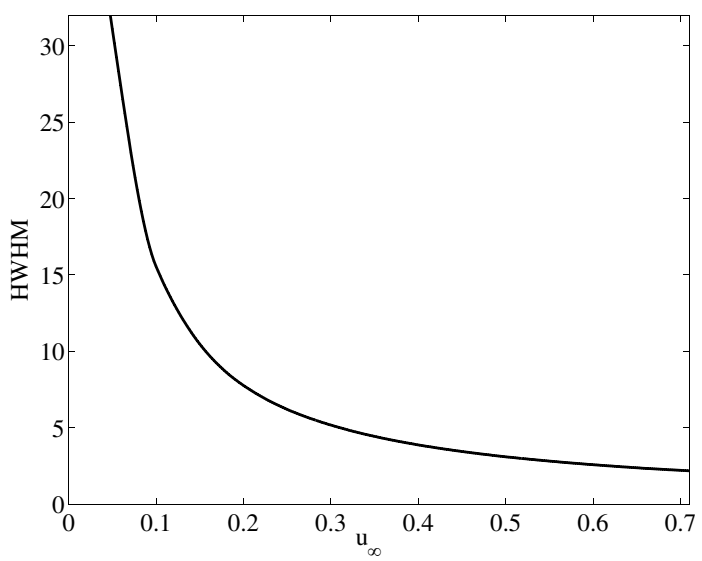

FIG. 4. Existence curve relating the normalized optical intensity HWHM of the soliton to $u_{\infty}$.

shows the existence of a normalized minimum HWHM $\simeq$ $2.1(\simeq 0.24 \lambda)$ for $u_{\infty}=1 / \sqrt{2}$.

It is interesting to examine the behavior of our solution in the limit of large $\rho$. To this end, neglecting in Eq. (15) the term in $u / \rho$, we have

$$
\frac{d^{2} u}{d \rho^{2}}=\left(u^{2}-u_{\infty}^{2}\right) u
$$

which formally coincides with the equation describing one dimensional linearly polarized paraxial dark solitons. Equation (21) admits of the solution $u=u_{\infty} \times$ $\tanh \left(\rho u_{\infty} / \sqrt{2}\right)$, which can be numerically compared with the exact solution of Eq. (12). The analysis shows that the hyperbolic tangent solution reproduces the exact one for large values of $\rho$, as expected, while it at most differs by a factor $\cong 1.2$ for small values of $\rho$.

Recent findings indicate that radially and azimuthally polarized laser beams can be efficiently generated $[17,18]$ and focused to subwavelength diameter [19] in resonatorlike schemes. These, combined with an appropriate nonlinear material, can form a basis for a first experimental investigation of the existence of the azimuthally polarized solitons. As an example, if we consider a strongly defocusing nonlinear medium, like sodium vapor at a density of $\sim 10^{12}$ atoms $/ \mathrm{cm}^{3}$ for which $n_{2}=-4 \times 10^{-14}(\mathrm{~m} / \mathrm{V})^{2}$ [20], the upper threshold for the asymptotic optical soliton intensity $I_{\infty}^{\max }$ is of the order of $.5 \mathrm{MW} / \mathrm{cm}^{2}$.

Finally, we wish to note that, for large values of $|u|^{2}$, other nonlinear contributions may become significant enough to affect the validity of Eq. (3). However, in our case, $|u|^{2}$ is limited by the upper value $1 / 2$ [see Eq. (14)] and these contributions are likely to be negligible. As an example, we can compare the standard cubic term $|u|^{2} u$ with the quintic one which can be written as $(1 / 2) \kappa^{2}|u|^{4} u$ (see Ref. [8]), where $\kappa=1 /(k w)$ ( $k$ and $w$ being the wave number and the beam width). The quintic term is obviously negligible when $(1 / 2) \kappa^{2}\left|u_{\infty}\right|^{2} \ll 1$, i.e., assuming $w \simeq \lambda / 4$, for $\left|u_{\infty}\right|^{2} \ll 5$.
In summary, we have found, to the best of our knowledge, the first exact, two-dimensional, dark spatial Kerr soliton. This soliton is obtained as a particular solution of an equation [Eq. (8)], which in turn exactly follows from Maxwell's equations, describing circularly symmetric, azimuthally polarized electric fields. Our $a b$ initio approach inherently overcomes any approximated scheme employed to describe paraxial or slightly nonparaxial situations. In particular, the derived analytical expression of the soliton propagation constant as a function of the normalized $(\rho \rightarrow \infty)$ field amplitude $u_{\infty}$ shows that the soliton does not exist whenever $u_{\infty}>1 / \sqrt{2}$. We have also numerically evaluated the existence curve relating the soliton width to $u_{\infty}$; as a relevant result, this curve shows that the soliton width attains the minimum possible value of about $\lambda / 4$ for $u_{\infty}$ approaching $1 / \sqrt{2}$.

This research has been funded by the Istituto Nazionale di Fisica della Materia through the "Solitons embedded in holograms", the FIRB "Space-Time nonlinear effects" projects and the Air Force Office of Scientific Research (H. Schlossberg).

*Electronic address: alessandro.ciattoni@aquila.infn.it

[1] S. Trillo and W. Torruellas, Spatial Solitons (Springer, Berlin, 2001).

[2] Y. Kivshar and B. Luther-Davis, Phys. Rep. 298, 81 (1998).

[3] M. D. Feit and J. A. Fleck, Jr., J. Opt. Soc. Am. B 5, 633 (1988).

[4] N. Akhmediev, A. Ankiewicz, and J. M. Soto-Crespo, Opt. Lett. 15, 411 (1993).

[5] G. Fibich, Phys. Rev. Lett. 76, 4356 (1996).

[6] A. P. Sheppard and M. Haelterman, Opt. Lett. 23, 1820 (1998).

[7] S. Chi and Q. Guo, Opt. Lett. 20, 1598 (1995).

[8] S. Blair, Chaos 10, 570 (2000).

[9] G. Fibich and B. Ilan, Physica D (Amsterdam) 157, 112 (2001).

[10] A. Ciattoni, C. Conti, E. DelRe, P. D. Porto, B. Crosignani, and A. Yariv, Opt. Lett. 27, 734 (2002).

[11] P. L. Kelley, Phys. Rev. Lett. 15, 1005 (1965).

[12] Y. Silberberg, Opt. Lett. 15, 1282 (1990).

[13] G. Fibich and A. L. Gaeta, Opt. Lett. 25, 335 (2000).

[14] B. Crosignani, A. Yariv, and S. Mookherjea, Opt. Lett. 29, 1254 (2004).

[15] A. Ciattoni, B. Crosignani, A. Yariv, and S. Mookherjea, (to be published).

[16] B. Crosignani, A. Cutolo, and P. Di Porto, J. Opt. Soc. Am. 72, 1136 (1982).

[17] R. Oron, S. Bilt, N. Davidson, A. A. Freisem, Z. Bomzon, and E. Hasman, Appl. Phys. Lett. 77, 3322 (2000).

[18] I. Moshe, S. Jackel, and A. Meir, Opt. Lett. 28, 807 (2003).

[19] R. Dorn, S. Quabis, and G. Leuchs, Phys. Rev. Lett. 91, 233901 (2003).

[20] G. A. Swartzlander, D. R. Andersen, J. J. Regan, H. Yin, and A. Kaplan, Phys. Rev. Lett. 66, 1583 (1991). 